OPEN ACCESS

Edited by:

Filippo Caraci,

University of Catania, Italy

Reviewed by:

Arianna Bellucci,

University of Brescia, Italy

Gaurav Bedse,

Vanderbilt University, United States

*Correspondence: Chihiro Tohda

chihiro@inm.u-toyama.ac.jp

Specialty section:

This article was submitted to Experimental Pharmacology and Drug

Discovery,

a section of the journal

Frontiers in Pharmacology

Received: 14 April 2017

Accepted: 19 May 2017

Published: 19 June 2017

Citation:

Yang Z, Kuboyama T and Tohda C

(2017) A Systematic Strategy

for Discovering a Therapeutic Drug

for Alzheimer's Disease and Its Target

Molecule. Front. Pharmacol. 8:340.

doi: 10.3389/fphar.2017.00340

\section{A Systematic Strategy for Discovering a Therapeutic Drug for Alzheimer's Disease and Its Target Molecule}

\author{
Zhiyou Yang, Tomoharu Kuboyama and Chihiro Tohda* \\ Division of Neuromedical Science, Department of Bioscience, Institute of Natural Medicine, University of Toyama, Toyama, \\ Japan
}

Natural medicines are attractive sources of leading compounds that can be used as interventions for neurodegenerative disorders. The complexity of their chemical components and undetermined bio-metabolism have greatly hindered both the use of natural medicines and the identification of their active constituents. Here, we report a systematic strategy for evaluating the bioactive candidates in natural medicines used for Alzheimer's disease (AD). We found that Drynaria Rhizome could enhance memory function and ameliorate AD pathologies in 5XFAD mice. Biochemical analysis led to the identification of the bio-effective metabolites that are transferred to the brain, namely, naringenin and its glucuronides. To explore the mechanism of action, we combined the drug affinity responsive target stability with immunoprecipitationliquid chromatography/mass spectrometry analysis, identifying the collapsin response mediator protein 2 protein as a target of naringenin. Our study indicates that biochemical analysis coupled with pharmacological methods can be used in the search for new targets for $A D$ intervention.

\footnotetext{
Keywords: Alzheimer's disease, axonal regeneration, CRMP2 phosphorylation, DARTS analysis, memory recovery, Drynaria rhizome
}

\section{INTRODUCTION}

The development of drugs from natural medicines is a powerful and promising research track. Because of the diversity of constituents in herbal medicines, multi-component interactions and multiple molecular targets must be considered, making it difficult to investigate the signaling mechanism. In vitro bioassay-guided isolation is one of the typical ways to access active compounds (Koehn and Carter, 2005) and provides a window into the mechanisms of herbal drugs. However, this approach does not always detect the actual active compounds because this method ignores the bio-metabolism of constituents before and after absorption (Wishart, 2016). The BBB permeability of these metabolites is one of the crucial factors in the development of a medicinal drug for central nervous system (CNS) diseases. In other words, the application of natural medicines to the treatment of neurodegenerative diseases is largely hindered by the limited knowledge of their metabolism, the effective metabolites and their brain transfer, and the molecular mechanisms underlying this metabolism. Therefore, addressing which metabolites are delivered to the brain is at the forefront of research to unravel the true mechanisms of natural medicines. 
Liquid chromatography-mass spectrometry (LC-MS) analysis has been a powerful tool for identifying micro constituents from the bio-samples obtained after the oral delivery of herbal drugs (Xiao et al., 2012). Based on LC-TOF/MS analyses, a few reports have shown selected brain-penetrating metabolites to be interventions for AD (Wang et al., 2012; Ho et al., 2013), but their targets and mechanisms were not well clarified. Our laboratory and others have shown that the drug affinity responsive target stability (DARTS) method is effective in identifying the target protein of a ligand (Lomenick et al., 2009; Tohda et al., 2012). Using this method, we explored the signaling of several small molecules (Tohda et al., 2012; Shigyo et al., 2015). In the present study, we established a series of procedures to investigate which proteins bind to the compounds and confirm the interaction using LC-MS and DARTS analyses to identify brain-active compounds after the oral administration of a drug.

Here, we construct a systematic strategy to unravel the mechanisms of action of the natural medicines used for $\mathrm{AD}$ treatment by exploring the brain-penetrating metabolites and their target proteins as a starting point. Briefly, the steps include (1) screening of effective natural medicines that may enhance axonal elongation and recover memory function using in vitro and in vivo models, which have been established previously (Tohda et al., 2004; Kuboyama et al., 2005); (2) systematically exploring effective metabolites delivered to the brain using chemical and pharmacological analyses; (3) identification of the target proteins as an initial point in identifying the signaling axis of effective metabolites; and (4) clarification of the specific signaling pathway driven by those identified metabolites. In this study, using DR as an example, we first found that DR was able to recover memory deficits in a murine $\mathrm{AD}$ model. By performing a systematic approach utilizing LTQ Orbitrap Fourier Transform Mass Spectrometry (LTQ-Orbitrap-FT-MS/MS), we identified real active metabolites that were distributed in the brain after the oral administration of DR. Furthermore, the target of the active metabolites was identified by a combination of DARTS and LTQ-Orbitrap-FT-MS/MS. We are the first to identify the effective form of $\mathrm{DR}$ and its mechanism for $\mathrm{AD}$ treatment.

\section{MATERIALS AND METHODS}

\section{Animal Studies}

All animal experiments were carried out in accordance with the Guidelines for the Care and Use of Laboratory Animals of the Sugitani Campus of the University of Toyama. All protocols were approved by the Committee for Animal Care and Use of the Sugitani Campus of the University of Toyama. The approval number for the animal experiments is A2014INM-1, and the confirmation number for the recombinant gene experiments is G2013INM-1.

Transgenic mice (5XFAD) were obtained from Jackson Laboratory (Bar Harbor, ME, United States). They were maintained as double hemizygotes by crossing with B6/SJL F1 breeders. To test the effect of the DR extract on 5XFAD mice, we used 5XFAD mice (male and female, 6-8 months old) and non-transgenic wild-type littermate mice (male and female, 6-8 months old). To investigate the effect of naringenin on 5XFAD mice, we used 5XFAD mice (male, 8-12 months old) and non-transgenic wild-type littermate mice (male, 8-12 months old). All mice were housed with free access to food and water and were kept in a controlled environment $\left(25 \pm 2^{\circ} \mathrm{C}, 12\right.$-h light/dark cycle starting at 7:00 am).

\section{Behavioral Test}

The DR extract was prepared as described previously (Yang et al., 2015). The DR extract was dissolved in saline to concentrations of $0.5,5$, and $50 \mathrm{mg} \mathrm{ml}^{-1}$. The DR extract or vehicle solution (saline) was orally administered once per day for 31 days. On day 20 of DR administration, mice were individually habituated to an open-field box composed of polyvinyl chloride $(33 \mathrm{~cm} \times 28 \mathrm{~cm}$; height, $26.5 \mathrm{~cm}$ ) for $10 \mathrm{~min}$. Their paths were tracked using a digital camera system. The distance moved for $10 \mathrm{~min}$ was considered the locomotor activity and was analyzed with Etho Vision 3.0 (Noldus, Wageningen, Netherlands). At days 21, 25, and 31 , the object recognition test (ORT), object location test (OLT), and episodic-like memory test (ELM) were performed as described previously (Kuboyama et al., 2015). Naringenin (Sigma-Aldrich, St. Louis, MO, United States) was suspended in saline to a concentration of 0.5 or $10 \mathrm{mg} \mathrm{ml}^{-1}$ and was orally administered once per day for 31 days. On days 21, 22, and 29 of naringenin administration, the open-field locomotion test, ORT, and OLT were performed.

\section{Immunohistochemistry}

After the behavioral studies, mice were deeply anesthetized with chloral hydrate (Wako, Tokyo, Japan) and perfused with saline followed by ice-cold $4 \%$ paraformaldehyde. Dissected brains were post-fixed in $4 \%$ paraformaldehyde for $24 \mathrm{~h}$ at $4^{\circ} \mathrm{C}$, immersed in $30 \%$ sucrose for $48 \mathrm{~h}$ for cryoprotection, and stored at $-30^{\circ} \mathrm{C}$. The brains were cut into $20 \mu \mathrm{m}$ coronal slices (six slices of each mouse brain) every $100 \mu \mathrm{m}$ in the perirhinal cortex area (bregma -1.46 to $-2.06 \mathrm{~mm}$ ) using a cryostat (Leica, Heidelberg, Germany). The slices were fixed with $4 \%$ paraformaldehyde and stained with a polyclonal antibody against $\mathrm{A} \beta(1-40 / 42)$ (1:300) (Chemicon, Temecula, CA, United States, cat \# AB5076), a monoclonal antibody against pNF-H (1:300) (Covance, Emeryville, CA, United States, cat \# SMI-35R), and a monoclonal antibody against PHF-tau (1:100) (Thermo Scientific, Rockford, IL, United States, cat \# MN1020) at $4^{\circ} \mathrm{C}$ for 24 h. Alexa Fluor 488-conjugated goat anti-rabbit IgG (1:300) and Alexa Fluor 594-conjugated goat anti-mouse antibody (1:300) were used as secondary antibodies (Molecular Probes, Eugene, OR, United States, cat \# ab150077 and ab150116, respectively). Fluorescence images of axons, PHF-tau and $\mathrm{A} \beta(1-40 / 42)$ were captured using a fluorescent microscope (BX-61/DP70, Olympus, Tokyo, Japan) at $324 \mathrm{~mm} \times 430 \mathrm{~mm}$. Six successive slices from the perirhinal cortex containing the hippocampus (CA1, CA3, and DG) of each mouse were captured for quantification. The images were analyzed with Image (NIH) as described previously (Tohda et al., 2012). 


\section{Bioavailability, Metabolism, and Brain Penetration of DR Extract}

The DR water extract was re-dissolved in $\mathrm{H}_{2} \mathrm{O}$ to a concentration of $10 \mathrm{mg} \mathrm{ml}^{-1}$ and filtered with a $0.45 \mu \mathrm{m}$ membrane; a $10 \mu \mathrm{l}$ sample was used for LC-MS analysis. To explore the metabolites that penetrated the brain, DR or vehicle solution (saline) was orally administered to 5XFAD mice (male and female, $n=3$ ) at a concentration of $13 \mathrm{~g} \mathrm{~kg}^{-1}$ for a single dose. Thirty minutes and $5 \mathrm{~h}$ after drug administration, mice were euthanized and blood was collected. The plasma was obtained after centrifugation of blood at $11,000 \mathrm{~g}$ for $10 \mathrm{~min}$ at $4^{\circ} \mathrm{C}$. The brain cortex was dissected following perfusion with saline. Plasma $(200 \mu \mathrm{l})$ was extracted with methanol, dried, and resolubilized in $50 \mu \mathrm{l}$ methanol. The brain cortex was homogenized and extracted with methanol, dried, and resolubilized in water (containing $0.1 \%$ formic acid) before loading onto preactivated Sep-Pak $\mathrm{C}_{18}$ SPE cartridges (Waters). The metabolites were eluted with methanol, vacuum dried, sonicated, and resolubilized in mobile phase before LC-MS analysis. To calculate the concentration of metabolites in the brain, the standard curve was made. Simply, the standard compounds were mixed with blank cortex and extracted as the method above, and then were applied to LC-MS analyses. A Thermo Scientific ${ }^{\text {TM }}$ Accela HPLC system interfaced with an LTQ Orbitrap XL hybrid Fourier Transform Mass Spectrometer (Thermo Fisher Co., San Jose, CA, United States) was used to chemically profile DR and the biosamples. Liquid chromatographic analysis was performed on a Capcell Pak $\mathrm{C}_{18}$ MGIII S-5 (1.5 mm i.d. $\times 150 \mathrm{~mm}$, Shiseido, Tokyo, Japan) column held at $40^{\circ} \mathrm{C}$ with a flow rate of $200 \mu \mathrm{l} / \mathrm{min}$. In the mobile phase, $0.1 \%$ aqueous formic acid (v/v) (A) and acetonitrile $(\mathrm{B})$ were used. The following linear elution gradient was used: $0-5 \mathrm{~min}, 6-20 \% \mathrm{~B} ; 5-10 \mathrm{~min}, 20-30 \% \mathrm{~B} ; 10-25 \mathrm{~min}$, $30-70 \% \mathrm{~B} ; 25-26 \mathrm{~min}, 70-6 \% \mathrm{~B} ; 26-32 \mathrm{~min}, 6 \% \mathrm{~B}$. The following ESI parameters were used: spray voltage $4.5 \mathrm{kV}$, capillary voltage $40.0 \mathrm{kV}$, tube lens $150 \mathrm{~V}$, capillary temperature $330^{\circ} \mathrm{C}$, sheath gas flow rate 50 units, and aux gas flow rate 10 units. We operated the mass spectrometer in the positive ESI mode, scanning from 50 to $2,000 \mathrm{~m} / \mathrm{z}$, and calibrated the instrument using a polytyrosine solution before each experiment.

\section{Primary Cultures and Measurement of Axonal Density}

Embryos were removed from a pregnant ddY mouse (SLC, Shizuoka, Japan) at 14 days of gestation as described previously (Tohda et al., 2012). The cells were treated with $10 \mu \mathrm{M} \mathrm{A} \beta_{25-35}$ or $5 \mu \mathrm{M} \mathrm{A} \beta_{1-42}$ (Sigma-Aldrich, A4559 or A9810) for 3 days before being treated with naringenin or vehicle (0.1\% DMSO) for 4 days. The $A \beta_{25-35}$ or $A \beta_{1-42}$ was previously incubated at $37^{\circ} \mathrm{C}$ for 4 or 7 days for aggregation, respectively. The cells were fixed with $4 \%$ paraformaldehyde and immunostained at $4^{\circ} \mathrm{C}$ for $24 \mathrm{~h}$ with a monoclonal antibody against pNF-H (1:500) as an axonal marker and a polyclonal antibody against MAP2 (1:2000, Abcam, Cambridge, United Kingdom, cat \# ab32454) as a neuronal marker. Alexa Fluor 488-conjugated goat anti-rabbit IgG (1:200) and Alexa Fluor 594-conjugated goat anti-mouse IgG (1:200) were used as secondary antibodies. Fluorescence images were captured using a fluorescence microscope system (BX61/DP70, Olympus) at $644 \mu \mathrm{m} \times 855 \mu \mathrm{m}$. The lengths of the pNF-H-positive axons were measured using a MetaMorph analyser (Molecular Devices, Sunnyvale, CA, United States).

\section{DARTS Analysis}

A mouse brain cortex was homogenized with M-PER (Thermo Scientific) containing $1 \times$ protease inhibitor cocktail (Thermo Scientific). The brain lysate $(2.7 \mathrm{mg}$ each) was added to $60 \mu \mathrm{l}$ of $6 \mathrm{mg} \mathrm{ml}^{-1} \mathrm{DR}$ extract or vehicle solution and incubated for $60 \mathrm{~min}$ at room temperature. The mixture was proteolysed using $2.7 \mathrm{mg}$ thermolysin (Sigma) in reaction buffer $[50 \mathrm{mM}$ Tris- $\mathrm{HCl}(\mathrm{pH} 8.0), 50 \mathrm{mM} \mathrm{NaCl}, 10 \mathrm{mM} \mathrm{CaCl}_{2}$ ] for $30 \mathrm{~min}$ at $37^{\circ} \mathrm{C}$. To stop the proteolysis, $0.5 \mathrm{M}$ EDTA ( $\mathrm{pH} 8.0$ ) was added to each sample in a 1:10 ratio. The samples $(600 \mu \mathrm{g}$ each) were then analyzed using 2D-PAGE. The separated proteins were stained using the colloidal Coomassie method. The spots that were thicker in the DR-treated lysate than the vehicletreated lysate were cut out and prepared for mass spectrometry analysis. Protein spots were excised, digested with trypsin (Promega, Madison, WI, United States), mixed with $\alpha$-cyano4-hydroxycinnamic acid in 50\% acetonitrile/0.1\% TFA, and subjected to MALDI-TOF analysis (Microflex LRF 20, Bruker Daltonics). DARTS and western blot analysis were performed to confirm the collapsin response mediator protein 2 (CRMP2) protein as a target of naringenin. A primary culture of mouse cortical neurons (ddY, E14) was maintained for $72 \mathrm{~h}$ without drug treatment. The cells were lysed, and cell lysate (15 $\mu \mathrm{g}$ each) was added to $1.67 \mathrm{ml}$ of $10 \mathrm{mM}$ naringenin in vehicle solution and incubated for $60 \mathrm{~min}$ at room temperature. The mixture was proteolysed using $3.75 \mathrm{ng}$ thermolysin in reaction buffer for $15 \mathrm{~min}$ at $37^{\circ} \mathrm{C}$. The reaction was stopped by adding 0.5 M EDTA ( $\mathrm{pH} \mathrm{8.0)} \mathrm{to} \mathrm{each} \mathrm{sample} \mathrm{at} \mathrm{a} \mathrm{1:10} \mathrm{ratio.} \mathrm{The} \mathrm{samples}$ were then subjected to SDS-PAGE for western blot analysis. Anti-CRMP2 (1:3000) (Sigma, St. Louis, MO, United States, cat \# C2993) and anti- $\beta$-actin (1:1000) (Cell Signaling Technology, cat \# 4967) antibodies were used as the primary antibodies, and HRP-conjugated goat anti-rabbit IgG (1:2000) (Santa Cruz, CA, United States, cat \# sc-2004) was used as the secondary antibody.

\section{IP Coupled with LC-MS Analysis}

Mouse primary cortical neurons (E14) were cultured for 3 days before $100 \mu \mathrm{M}$ naringenin was added for $0.5,2$, or $6 \mathrm{~h}$. After being washed three times with $1 \times$ PBS, neuronal cells were lysed with M-PER solution. Thirty micrograms of protein from each sample was denatured and extracted with $100 \mu \mathrm{l}$ methanol, and the supernatant was subjected to LC-MS after centrifugation at $14,000 \mathrm{~g}$ for $10 \mathrm{~min}$. For immunoprecipitation (IP), the lysates $(30 \mu \mathrm{g})$ treated with naringenin for $6 \mathrm{~h}$ were incubated with antibody (CRMP2 or IgG, $3.5 \mu \mathrm{g}$ ) for $1 \mathrm{~h}$ before being incubated with protein $G$ dynabeads (Life Technologies) for $10 \mathrm{~min}$ at $4^{\circ} \mathrm{C}$. The beads were washed three times with PBS containing $0.02 \%$ Tween 20 and then extracted with methanol. The supernatant was applied to LC-MS after centrifugation for $10 \mathrm{~min}$ at $14,000 \mathrm{~g}$. The LC-MS conditions were the same as those in the aforementioned experiment except for the linear elution 
gradient: $0-5 \mathrm{~min}, 20-50 \% \mathrm{~B}$; 5-20 $\mathrm{min}, 50-90 \% \mathrm{~B} ; 20-21 \mathrm{~min}$, 90-20\% B; 21-27 min, 20\% B.

\section{SiRNA Knockdown}

siRNAs were transfected into mouse cortical neurons (ddY E14) together with GFP vectors according to the manufacturer's protocol for nucleofection (Lonza, Basel, Switzerland). Briefly, mouse cortical neurons $\left(5.0 \times 10^{6}\right.$ cells $)$ were mixed with siCRMP2 ( $1 \mu \mathrm{M}$, Thermo Scientific, cat \# 4390771) or control siRNA (1 $\mu \mathrm{M}$, Thermo Scientific, 4390843) and GFP vector (2 $\mu \mathrm{g}$, Lonza, Basel, Switzerland) and electroporated with an Amaxa Nucleofector (Lonza). Three days after the transfection, cells were fixed and immunostained with a polyclonal antibody against CRMP2 (Sigma, cat \# C2993). Double immunostained neurons were selected for statistical analysis. The appropriate concentration of siRNA for knockdown was determined previously. Three days after the transfection of siRNA, the cells were treated with naringenin $(10 \mu \mathrm{M})$ or vehicle solution (0.1\% DMSO). Four days after treatment, the axon density was measured.

\section{In Vitro Evaluation of Phosphorylated CRMP2}

Mouse primary cortical neurons (E14) were cultured for 3 days and then treated with $10 \mu \mathrm{M} \mathrm{A} \beta_{25-35}$ for another 3 days. After removal of the $A \beta$-containing medium, the cells were treated with naringenin $(1 \mu \mathrm{M})$ for $0.5,24$, or $96 \mathrm{~h}$. Cells were fixed and double immunostained with a polyclonal antibody against pThr514 CRMP2 (1:200) (Cell Signaling Technology, cat \# 9397) and a monoclonal antibody against MAP2a/2b (1:200, Fremont, CA, United States, cat \# MS-249-S) as a neuron marker. The expression of pCRMP2 was measured using a CS Analyzer (ATTO, Tokyo, Japan).

\section{Statistical Analysis}

Graphs were generated with GraphPad Prism 5 (GraphPad Software, La Jolla, CA, United States), and Student's unpaired $t$-test, one-way analysis of variance (ANOVA) with Dunnett's post hoc test and two-way ANOVA with Bonferroni post hoc test were performed for statistical analysis of the data, and the statistical significance criterion $P$-value was 0.05 . The data are presented as the mean \pm SEM.

\section{RESULTS}

\section{DR Extract Ameliorates Memory Deficits in the 5XFAD Mouse Model}

DR extracts $\left(5,50\right.$, or $500 \mathrm{mg} \mathrm{kg}^{-1}$ per day) or vehicle solutions were orally administered to 5XFAD mice. The determination of DR doses was according to the animal study of other groups (Anuja et al., 2010; Lee et al., 2014). The memory test scheme was showed in Figure 1A. In the object recognition memory test (Figure 1C), all of the mice showed equivalent exploratory behavior toward each of the objects (preference indices were approximately 50\%) in the training session. In the test session, wild-type mice and DR (50 and $500 \mathrm{mg} \mathrm{kg}^{-1}$ per day)-treated 5XFAD mice exhibited significantly more frequent exploratory behavior toward a novel object than vehicle-treated 5XFAD mice (approximately 50\%). Improvement of recognition memory by DR (500 $\mathrm{mg} \mathrm{kg}^{-1}$ per day) was confirmed by other independent experiments, too (Supplementary Figures 1A,B).

To test whether spatial memory is improved by DR, we performed the object location memory test (Figure 1D); in this test, in which one of the novel objects was located in a different place in the test session, the vehicle-treated 5XFAD mice could not distinguish the object with the novel location from the object with the familiar location, whereas wild-type mice and DR (500 mg kg-1 per day)-treated 5XFAD mice significantly increased their exploratory behavior toward the object in the novel location. The ELM was carried out to evaluate the ability of the mice to integrate object (what), location (where), and context recognition (when) (Dere et al., 2005). In this test, among the four objects in the test session, E1 and E4 were located at the same places in training session 2, while the objects D2 and D4' had been presented to mice in training session 1 (Figure 1E(1)). Therefore, objects D2 and D4' were more unfamiliar than objects E1 and E4 in the test session. Wild-type mice and DR (50 and $500 \mathrm{mg} \mathrm{kg}^{-1}$ per day)-treated 5XFAD mice significantly increased their exploratory behavior toward the unfamiliar objects (D2 and D4') compared with the familiar objects (E1 and E4) (Figure 1E(2)). The vehicle-treated $5 \mathrm{XFAD}$ mice failed to distinguish between the presented objects. For objects D2 and D4, D2 was presented to mice at the same location in training session 1. D4' was located at a novel place for the mice; therefore, if the mouse memorized object places and presentation orders, the mouse would show higher preference indices for object D4' than D2. Like the vehicle-treated 5XFAD mice, the wild-type mice failed to recognize objects D2 and D4'; in contrast, DR (500 $\mathrm{mg} \mathrm{kg}^{-1}$ per day)-treated 5XFAD mice significantly increased their exploratory behavior toward object D4' rather than D2 (Figure 1E(3)), this might due to the memory improvement of DR on normal mice (Supplementary Figures $2 \mathrm{~A}-\mathrm{C}$ ). The animals' body weights were recorded during the drug administration, with no significant changes observed in any of the groups (Supplementary Figure 2D). In the open-field test, no significant locomotor differences were detected in the distances moved and velocity among the five groups (Figure 1B and Supplementary Figure 2E).

Collectively, these results indicate that DR extract rescues cognitive deficits when orally administered to 5XFAD transgenic mice.

\section{DR Extract Ameliorates AD-Like Pathology in 5XFAD Mice}

All of the aforementioned wild-type mice and 5XFAD mice were euthanized after the behavior tests. To examine the effect of orally administered DR on the number of $A \beta$ plaques, the brains of mice were sectioned and stained for $A \beta_{1-42}$ to visualize $\mathrm{A} \beta$ plaques. We measured $\mathrm{A} \beta$ plaques in the perirhinal cortex and hippocampus of the brain. Amyloid deposits were primarily localized in the cerebral cortex and hippocampus of 5XFAD mice, 


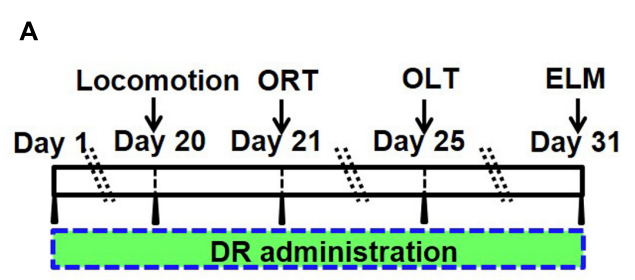

C

Object recognition test (ORT)
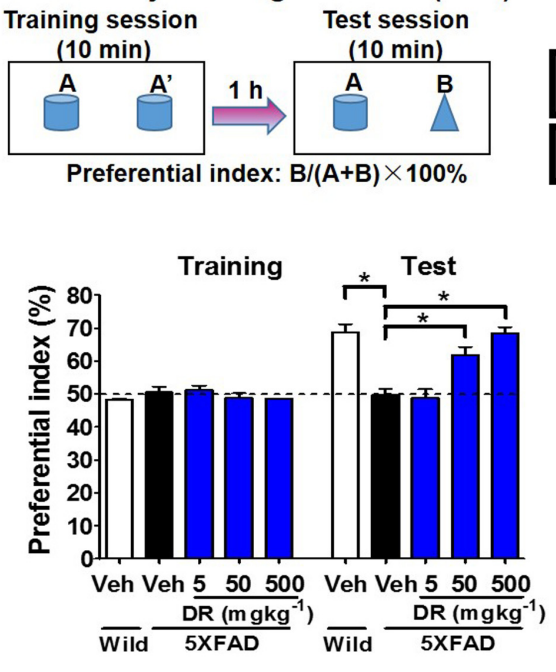

B $\bar{\varepsilon}$

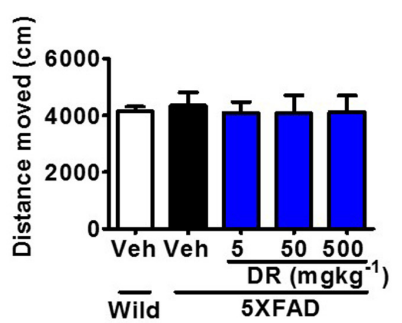

D

Object location test (OLT)

Training session Test session

$(10 \mathrm{~min}) \quad(10 \mathrm{~min})$

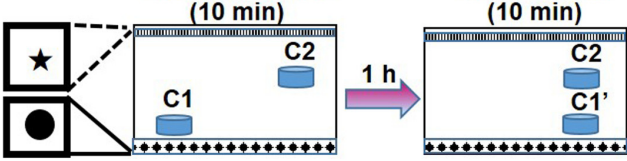

Preferential index: $\mathrm{C}^{\prime} /\left(\mathrm{C} 1^{\prime}+\mathrm{C} 2\right) \times 100 \%$

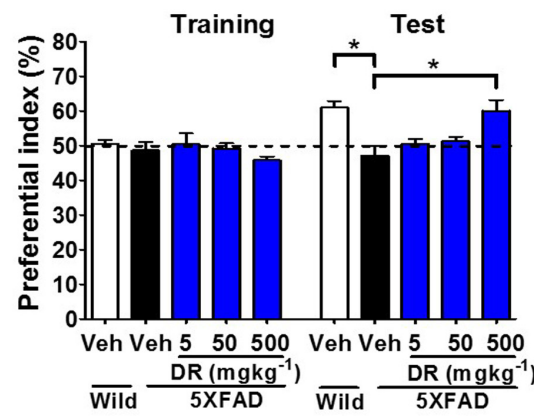

$\mathbf{E}$

(1)

Episodic-like memory test (ELM)
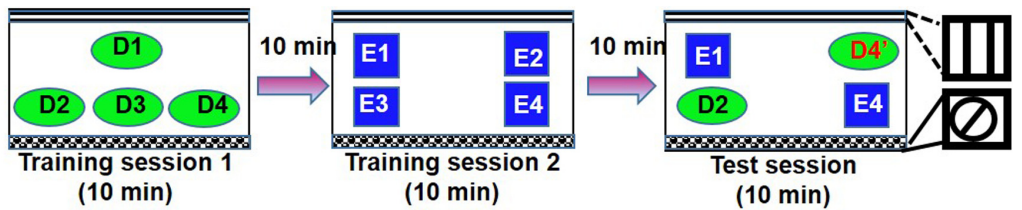

(2)

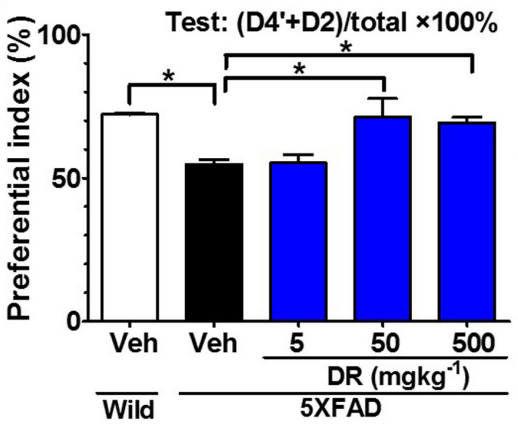

(3)

D2/total $\times 100 \% \square \mathrm{D} 4 /$ total $\times 100 \%$

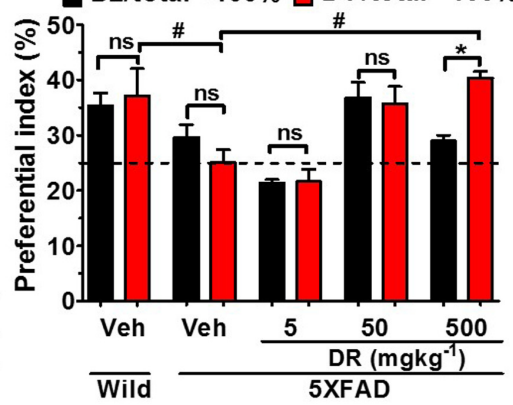

FIGURE 1 | DR extract rescues memory deficits in 5XFAD mice. (A) Time course of the experiments. (B-E) Effects of DR extract on the open-field test, object recognition memory, object location memory, and episodic-like memory. Six- to eight-month-old wild-type mice and 5XFAD mice were used ( $n=3-5)$. (B) Total distance moved (within $10 \mathrm{~min}$ ) in the open-field test. (C) Object recognition test. The preference indices for objects A' (training session) and B (test session) were quantified. $p<0.0001$, drug $\times$ time interaction was analyzed using repeated measures two-way ANOVA, $F(4,15)=13.18 .{ }^{*} p<0.05$, post hoc Bonferroni test. (D) Object location test. The preference indices for objects C1 (training session) and C1' (test session) were quantified. $p=0.007$, drug $\times$ time interaction was analyzed using repeated measures two-way ANOVA, $F(4,15)=5.34 .{ }^{*} p<0.05$, post hoc Bonferroni test. (E) Episodic-like memory test. (E1) Time schedule of ELM. (E2) The preference indices for objects D4' plus D2 (test session) were quantified. ${ }^{*} p<0.05$, one-way ANOVA post hoc Bonferroni test. (E3) The preference indices for objects D2 (black columns) and D4' (red columns) were also quantified. ${ }^{*} p<0.05$, two-way ANOVA post hoc Bonferroni test. * $p<0.05$, one-way ANOVA post hoc Bonferroni test. 

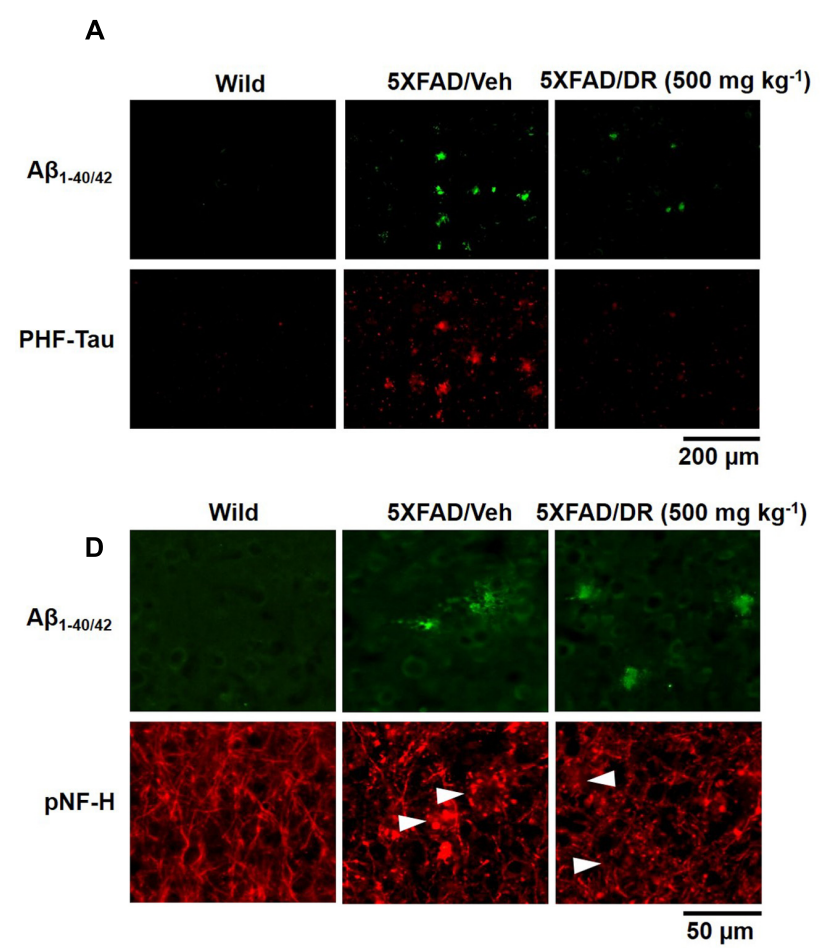

B
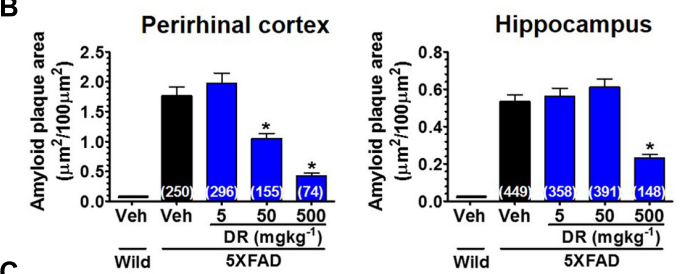

C
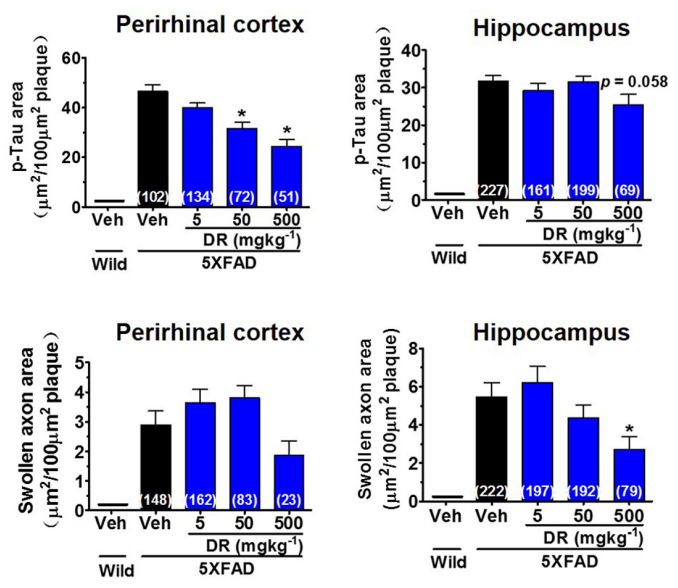

FIGURE 2 | DR extract ameliorates AD-like pathology in 5XFAD mice. DR extract (5,50,500 mg kg ${ }^{-1}$, p.o.) or vehicle solution (saline) was administered to wild-type or 5XFAD mice (males and females, 6-8 months old) for 31 days. The day after the behavioral test, mice were sacrificed and subjected to brain analysis.

(A) Representative images of $A \beta_{1-40 / 42}$-positive plaques and PHF-tau in the perirhinal cortex. (B) The total area of amyloid plaques per $100 \mu m^{2}$ was quantified in the cerebral cortex and hippocampus. (C) The total area of PHF-tau associated with A plaques per $100 \mu \mathrm{m}^{2}$ was quantified in the cerebral cortex and hippocampus. (D) $A \beta$ plaques and axons were double immunostained with $A \beta_{1}-40 / 42$ and pNF-H antibodies. pNF-H-positive bulb- or ring-like axonal structures were localized with A $\beta$ plaques. Representative images from the perirhinal cortex are shown. (E) The total area of abnormal axons per $100 \mu m^{2}$ of amyloid plaque in the cerebral cortex and hippocampus are shown. One-way ANOVA and Dunnett's post hoc test were used for all statistical analyses $\left(^{*} p<0.05\right.$ vs. veh/5XFAD; the number of measured areas is shown in each column).

while no $\mathrm{A} \beta$ deposits were observed in the age-matched wild-type mice (Figure 2A and Supplementary Figure 2F). DR treatment dose-dependently reduced $A \beta$ plaques in the cortex of 5XFAD mice and diminished $A \beta$ plaques in the hippocampus at the $500 \mathrm{mg} \mathrm{kg}^{-1}$ per day dose (Figure 2B).

To assess the level of paired helical filament (PHF)-tau, another key neuropathological feature in $\mathrm{AD}$, we stained the brain slices with an anti-phosphorylated tau antibody. PHF-tau was observed very close to the $\mathrm{A} \beta$ plaques (Figure 2A) in 5XFAD mice, while no PHF-tau was observed in the wild-type mice. DR dose-dependently decreased the levels of PHF-tau in the cortex, and a decreasing trend in PHF-tau was observed in the hippocampus after DR treatment (Figure 2C).

As DR stimulated axonal outgrowth after $A \beta_{25-35}$-induced atrophy in cultured primary cortical neurons (Yang et al., 2015), we investigated whether any changes were induced by DR treatment in 5XFAD mice. Abnormal axon structures, such as bulb- or ring-like neurofilaments stained with pNF-H, are located within amyloid plaques in both preclinical and end-stage $\mathrm{AD}$ patients (Dickson and Vickers, 2001). Our previous study also indicated that bulb-shaped axons are colocalized with amyloid plaques in 5XFAD mice (Tohda et al., 2012). The axons in the vehicle-treated 5XFAD mice were blocked at the front of amyloid plaques and formed swollen bulb- or ring-like shapes (Figure 2D). Abnormal axons were rarely detected in the distal part of amyloid plaques. DR (500 $\mathrm{mg} \mathrm{kg}^{-1}$ per day) treatment significantly reduced the swollen axonal areas in the brain cortex and hippocampus (Figure 2E). Collectively, these results indicate that orally administered DR effectively repairs degenerated axons in the brains of 5XFAD mice, which might contribute to the recovery of memory function.

\section{DR-Derived Metabolites Penetrate the BBB after the Oral Administration of DR Extracts to 5XFAD Mice}

To clarify the mechanism through which DR may function in treating $\mathrm{AD}$, we tried to detect DR-derived metabolites that are delivered into the brain. Using the high-accuracy quasi-molecular ion $\left([\mathrm{M}+\mathrm{H}]^{+}\right)$and a mass error of $\pm 1 \mathrm{mmu}$, we comprehensively characterized 17 compounds by comparing their MS-MS data and fragmentation patterns with those of reference standards or reported compounds (Supplementary Figure 3 and Table 1). Naringin and neoeriocitrin are the two main compounds in DR extract, with contents of 96.8 and $108.0 \mathrm{mg} \mathrm{g}^{-1}$, respectively. Naringenin was present at $0.27 \mathrm{mg} \mathrm{g}^{-1}$ in DR extract. Naringenin 


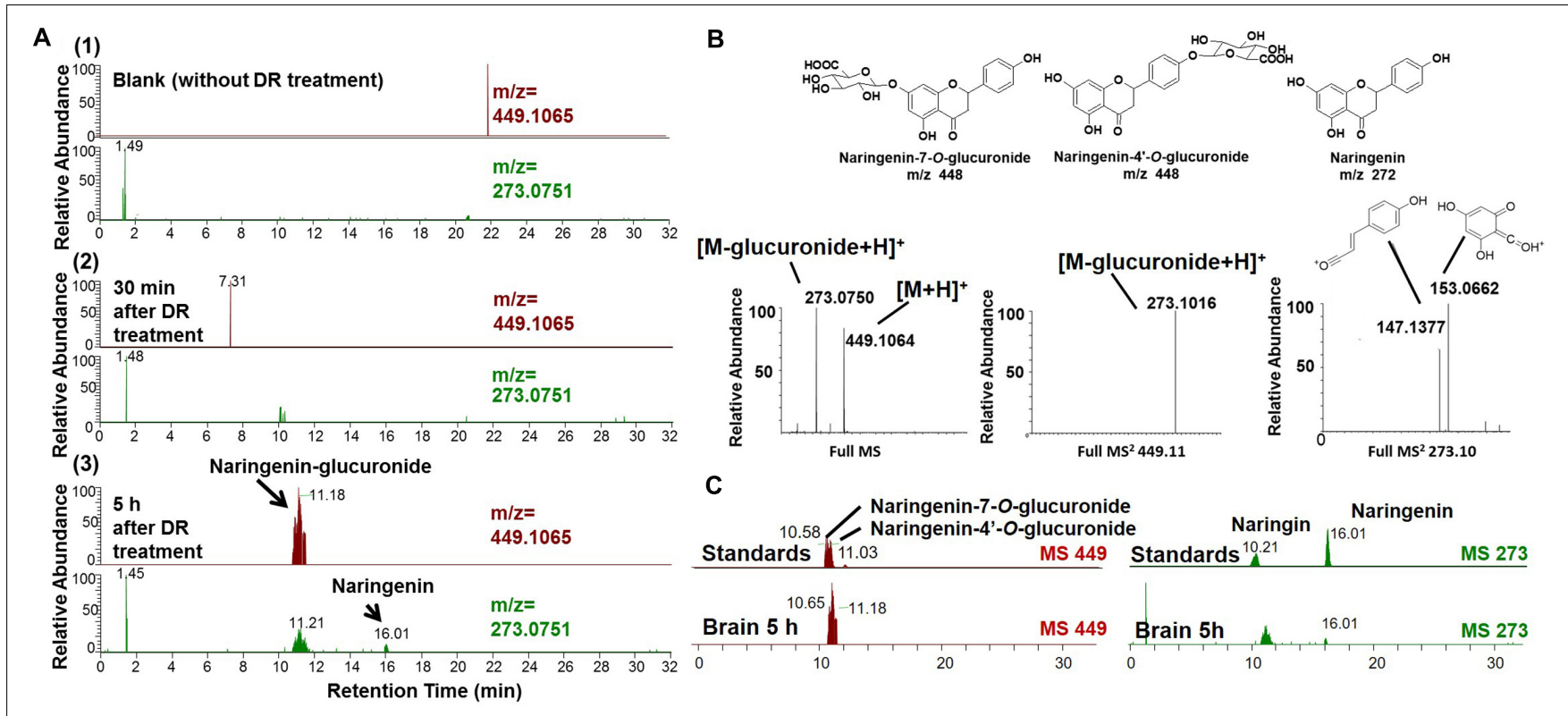

FIGURE 3 | Brain-penetrating metabolites after the oral administration of DR extracts to 5XFAD mice. DR extract (13 $\mathrm{g} \mathrm{kg}^{-1}$, p.o.) or vehicle solution (saline) was administered to mice (male and females, 12 months old, $n=3$ ) as a single dose. Mice were sacrificed 0.5 or $5 \mathrm{~h}$ after treatment, and the brain cortex was extracted with methanol and subjected to LC-MS analysis. (A) Extracted ion current (EIC) chromatograms of naringenin glucuronide $(\mathrm{m} / \mathrm{z}=449.1065)$ and naringenin ( $\mathrm{m} / \mathrm{z}=273.0751)$. (A1) Without DR treatment; (A2) $30 \mathrm{~min}$ after DR treatment; (A3) $5 \mathrm{~h}$ after DR treatment. (B) Proposed structures of the DR metabolites identified as naringenin-7/4'-O-glucuronide and naringenin (Upper) and mass fragmentation characteristics of naringenin glucuronide (Lower). (C) Comparison of the ElC chromatograms for naringenin-7/4'-O-glucuronide, naringenin, and naringin standards and samples obtained $5 \mathrm{~h}$ after DR treatment.

and naringenin glucuronide are metabolized from naringin and were detected in the plasma of rats after the oral administration of naringin (Fang et al., 2006). Then, we profiled the chemicals that were delivered into the blood and brain in 5XFAD mice with HPLC-FT-MS. Usually higher doses than effective doses are selected to detect the metabolites of herbal extracts (Xiang et al., 2011), formulations (Fujiwara et al., 2011; Kushida et al., 2013), and even single compounds (Durairajan et al., 2012; Tan et al., 2013) in biosamples. Therefore, we orally administered a high dose of DR $\left(13 \mathrm{~g} \mathrm{~kg}^{-1}\right)$ to the 5XFAD mice. Using SIEVE software (Niu et al., 2015), we performed differential analyses of the total ion current (TIC) chromatograms for the biosamples from vehicle-treated and DR-treated mice (Supplementary Figure 4). The different TIC peaks were analyzed and considered based on their retention times, accurate mass values, and MS-MS fragment patterns. Thirteen compounds or metabolites were detected in the plasma of DR-treated 5XFAD mice, and 3 chemicals were detected in the brain cortex of DR-treated 5XFAD mice (Supplementary Table 1). The extracted ion current (EIC) chromatograms of the speculated brain-delivered metabolites are shown in Figure 3A. These metabolites were detected in the brains $5 \mathrm{~h}$ after administration of DR (Figure $3 \mathrm{~A}(3)$ ) but not detected in the brains of vehicle-treated SXFAD mice (Figure $3 \mathbf{A}(\mathbf{1})$ ) or $30 \mathrm{~min}$ after the oral administration of DR extract (Figure 3A(2)). To confirm the structures of the speculated metabolites, we synthesized naringenin-7-Oglucuronide and naringenin- $4^{\prime}-O$-glucuronide from naringenin and analyzed the mass fragmentation characteristics of these compounds (Figure 3B). The HPLC-MS results showed similar retention time and MS-MS fragmentations in the standards and DR-treated mouse brain samples (Figure 3C). Our preexperiment showed that the $C_{\max }$ of metabolites was $5 \mathrm{~h}$ after DR administration. Using the standard curve, the calculated levels of naringenin, naringenin-7-O-glucuronide, and naringenin- $4^{\prime}-O-$ glucuronide metabolite in the brain are $15.1 \pm 2.9,302.6 \pm 77.9$, and $22.7 \pm 17.8 \mathrm{pmol} \mathrm{g}^{-1}$ following DR treatment, respectively. Taken together, these results suggest that the metabolites naringenin and glucuronides of naringenin at the $4^{\prime}$ or 7 position can penetrate the $\mathrm{BBB}$ after the oral administration of $\mathrm{DR}$ extract.

\section{DR-Derived Brain Penetrating Metabolites Enhance Axonal Growth in Cultured Neurons after $A \beta$ Treatment}

To test the effects of brain-penetrating metabolites on axonal growth, we investigated their effects on both $A \beta_{1-42}$ and $\mathrm{A} \beta_{25-35}$-induced axonal atrophy in cultured cortical neurons. According to the bioavailability of DR $\left(13 \mathrm{~g} \mathrm{~kg}^{-1}\right)$ in vivo, we calculated the total accumulated naringenin metabolites in the brain at a concentration of $13.1 \mathrm{nM}$ after DR (500 $\mathrm{mg} \mathrm{kg}^{-1}$ ) administration. Therefore, we selected the concentration of metabolites from 10 to $10 \mu \mathrm{M}$ for the study in vitro. Axonal density was decreased significantly by $\mathrm{A} \beta_{25-35}$ or $A \beta_{1-42}$ treatment, whereas treatment with naringenin $(1 \mu \mathrm{M})$ and naringenin-7-O-glucuronide $(0.1 \mu \mathrm{M})$ significantly increased the length of pNF-H (phosphorylated neurofilament $\mathrm{H}$, axonal marker)-positive axons (Figures $4 \mathrm{~A}, \mathbf{B}$ ). What's more, 
A

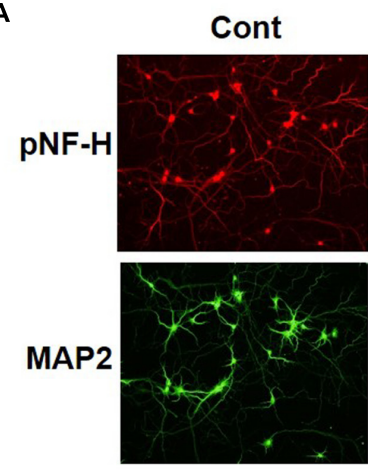

B
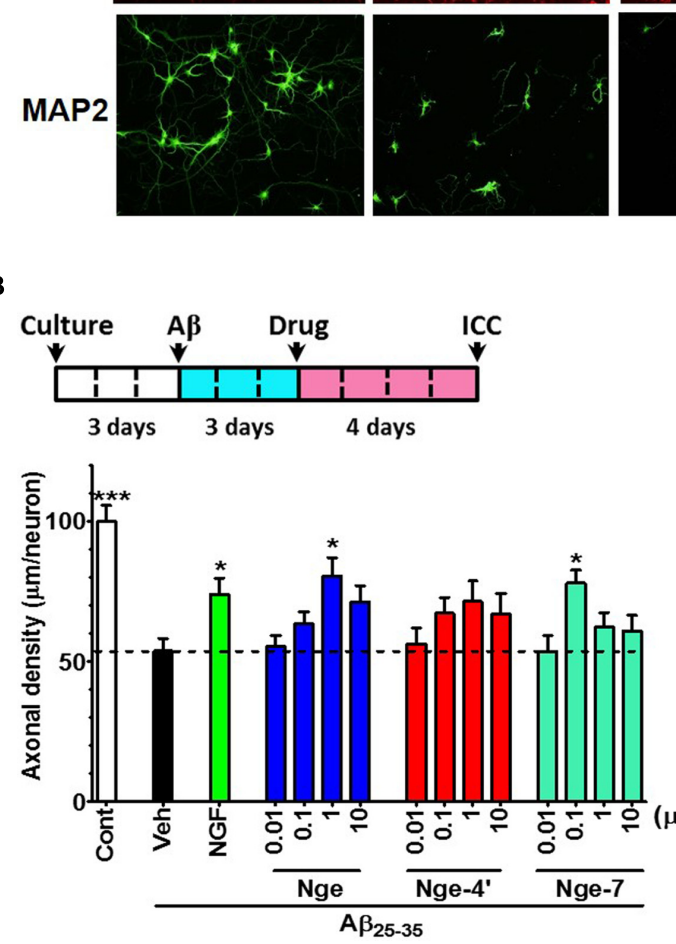

A $\beta /$ NGF
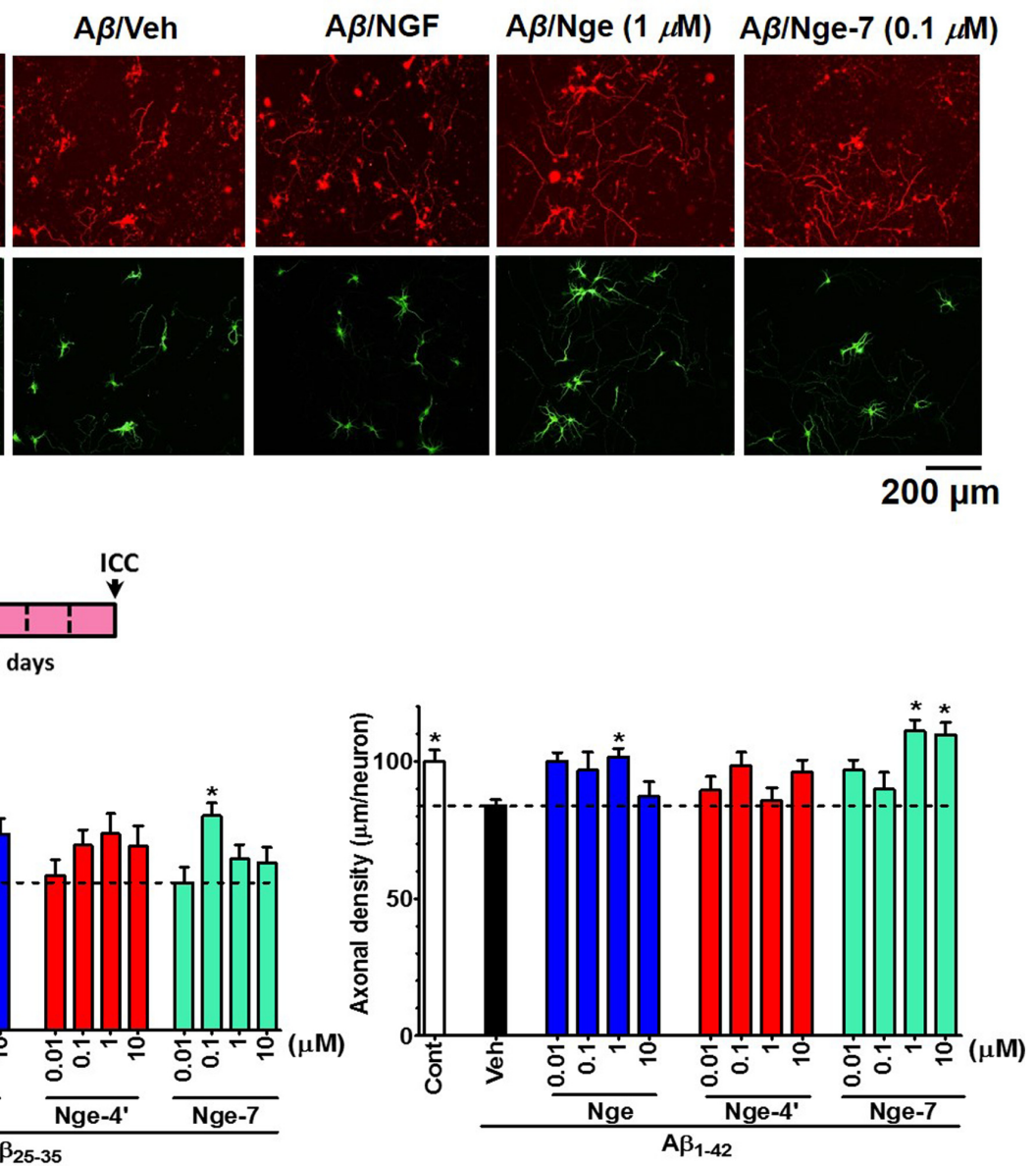

FIGURE 4 | Effects of the metabolites naringenin-7/4'-O-glucuronide (Nge-7 or Nge-4') and naringenin (Nge) on axonal regrowth after A $\beta$-induced axonal atrophy. Primary cortical neurons were cultured for 3 days, and then the cells were treated with $10 \mu \mathrm{M} \mathrm{A} \beta_{25-35}$ or $5 \mu \mathrm{M} A \beta_{1-42}(\mathrm{Veh})$. Three days later, the $\mathrm{A} \beta$ was removed and one of the compounds $(0.01,0.1,1$, and $10 \mu \mathrm{M})$, NGF $(100 \mathrm{ng} / \mathrm{ml})$, or vehicle $(0.1 \% \mathrm{DMSO})$ was added to the neurons. Four days after the treatment, the neurons were fixed and immunostained for pNF-H and MAP2. The lengths of the pNF-H positive neurites were measured. (A) Representative images of pNF-H-positive axons and MAP2-positive neurons in A $\beta_{25-35}$ treated group. Scale bar, $200 \mu \mathrm{m}$. (B) Time course of the experiments (Upper). Quantification of total axonal outgrowth and associated statistics (Lower). The error bars represent SEM. One-way ANOVA and Dunnett's post hoc test were performed (* $p<0.05$ vs. A $\beta$ Neh; $n=10-40$ photographs).

naringenin $(1 \mu \mathrm{M})$ and naringenin-7-O-glucuronide (1 and $10 \mu \mathrm{M})$ significantly reversed $\mathrm{A} \beta_{1-42}$ induced axonal atrophy (Figure 4B). NGF was used as a positive control for axonal extension after $A \beta_{25-35}$ treatment (Kuboyama et al., 2005). This result indicates that naringenin and naringenin-7-O-glucuronide can restore $\mathrm{A} \beta$-induced axonal atrophy.

\section{Naringenin Ameliorates Memory Deficits and AD-Like Pathology in 5XFAD Mice}

According to the contents of naringin and naringenin in DR extract, and naringin could be metabolized to naringenin in vivo according to our experiment and other reports. We calculated the total amount of naringenin aglycone in DR extract. Twenty-three milligram naringenin is contained in $500 \mathrm{mg}$ DR extract. We also referred to the dose of naringenin in reports of other AD models (Yang et al., 2014; Ghofrani et al., 2015). We orally administered naringenin (5 and $100 \mathrm{mg}$ $\mathrm{kg}^{-1}$ per day) to the 5XFAD mice (male, 8-12 months old) for 31 days (Figure 5A). In the ORT (Figure 5C), wild-type mice and naringenin $100 \mathrm{mg} \mathrm{kg}^{-1}$ per day-treated 5XFAD mice exhibited significantly more frequent exploratory behavior toward the novel object than vehicle-treated 5XFAD mice. In the OLT, naringenin $100 \mathrm{mg} \mathrm{kg}^{-1}$ per day-treated 5XFAD mice showed the trend of increased object location memory (Supplementary Figure 5B). There were no significant differences in the distances moved by the four groups in the open-field test (Figure 5B). During drug administration, the animals' body weights did not change significantly (Supplementary Figure 5A).

To examine the effect of orally administered naringenin on AD-like pathology in 5XFAD mice, we performed immunohistochemistry using antibodies against $\mathrm{A} \beta_{1-42}$ and PHF-Tau. As shown in Figure 5D, no amyloid plaques, PHF-tau, or degenerated axons were observed in the wild-type 
A

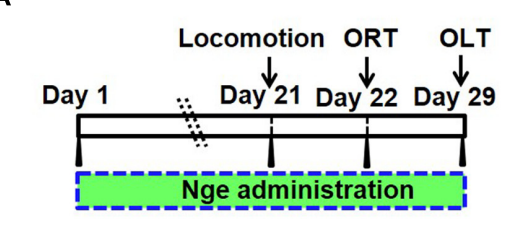

D
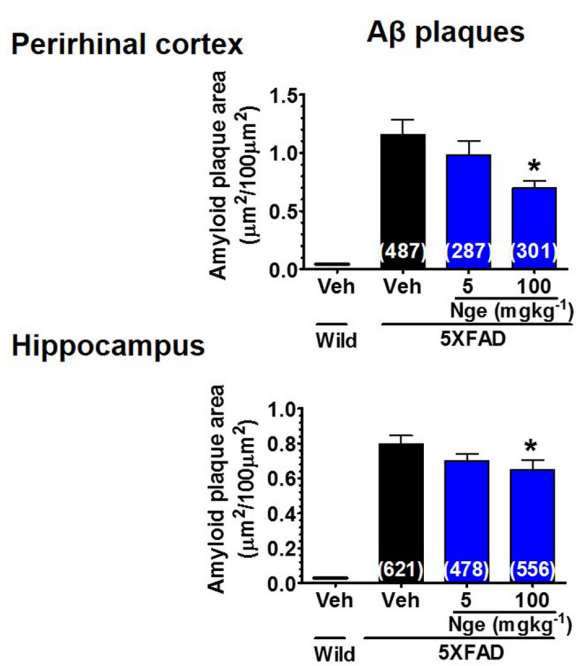

B

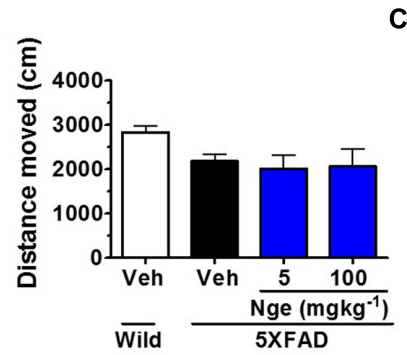

P-Tau associated with plaques

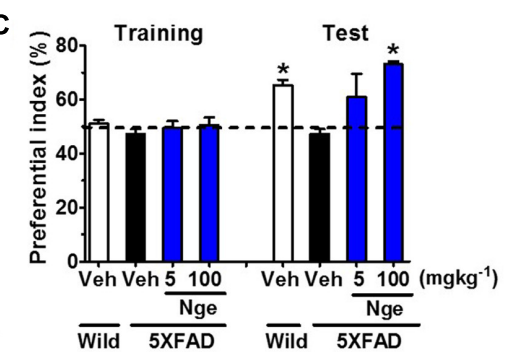

Swollen axons associated with plaques
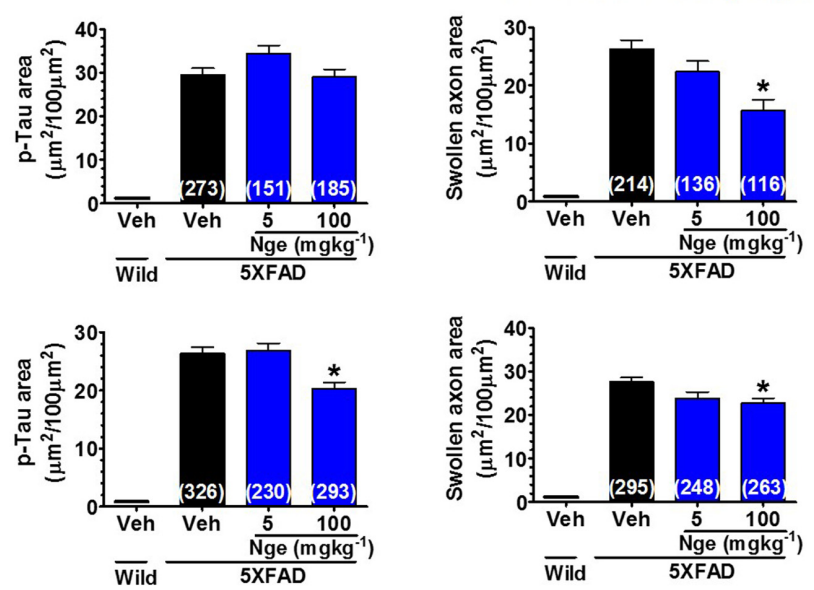

FIGURE 5 | Naringenin ameliorates recognition memory deficits and AD pathologies in 5XFAD mice. (A) Time course of behavioral tests. Naringenin (0, 5, or 100 mg $\mathrm{kg}^{-1}$ per day) was orally administered to 5XFAD mice (male, 8-12 months old, $n=4-5$ ) for 29 days, and their behavioral performance was evaluated. (B) Total distance moved (within $10 \mathrm{~min}$ ) in the open-field test. (C) Object recognition test. The preferential indices for the training and test sessions are shown. $p=0.009$, drug $\times$ time interaction was analyzed using repeated measures two-way ANOVA, $F(3,11)=6.24 .{ }^{*} p<0.05$ vs. vehicle-treated 5XFAD mice, post hoc Bonferroni test. (D) The total area of amyloid plaques, PHF-tau associated with amyloid plaques, and swollen axons associated with amyloid plaques were measured in the perirhinal cortex and hippocampus. One-way ANOVA and Dunnett's post hoc test were performed in all statistical analyses $\left({ }^{*} p<0.05\right.$ vs. vehicle-treated $5 X F A D$ mice).

mice. The naringenin (100 $\mathrm{mg} \mathrm{kg}^{-1}$ per day)-treated 5XFAD mice exhibited a significant reduction in amyloid plaques and swollen axon expressions (associated with the plaques) in the perirhinal cortex and hippocampus. The PHF-tau expression in the hippocampus (associated with the plaques) was significantly decreased by naringenin (100 $\mathrm{mg} \mathrm{kg}^{-1}$ per day) treatment. Taken together, the findings revealed that orally administered naringenin ameliorates memory deficits and effectively decreases amyloid plaques, plaque-associated PHF-tau and swollen axons in 5XFAD mice, as shown for DR administration.

\section{Naringenin Targets CRMP2 and Reduces the Phosphorylation of CRMP2 in Cultured Primary Neurons}

To explore the mechanism responsible for naringenin-induced axonal growth and memory improvement, we first used the DARTS method (Lomenick et al., 2009; Pai et al., 2015) to identify a target protein of naringenin. When a compound binds to a target protein, the protein conformation is probably changed, changing the protease sensitivity of the target protein
(Lomenick et al., 2009). Based on our previous study, a 62$\mathrm{kDa}$ protein spot was thicker in the DR-treated brain lysate than the vehicle on a 2D-PAGE gel after thermolysin reaction (Supplementary Figure 6), MALDI-TOF-MS analysis indicated that the spot was possibly CRMP2. To confirm CRMP2 as a target protein of naringenin, we performed DARTS and western blot analyses using cell lysates from mouse primary cultured cortical neurons (Figure 6A). After thermolysin treatment, the CRMP2 level in naringenin-treated lysates was significantly decreased compared with the vehicle solution-treated group (Figure 6B and Supplementary Figure 9).

Next, to confirm the binding of naringenin to CRMP2, we performed LC-MS combined with IP analysis. First, we evaluated whether naringenin could be taken up by the neurons. We found that naringenin accumulated in the neurons in a timedependent manner (Supplementary Figures 7A,B). Surprisingly, naringenin glucuronides were also detected in the neurons 30 min after incubation (Supplementary Figure 7A). Then, lysates were prepared from neurons after $6 \mathrm{~h}$ of incubation with naringenin and were subjected to IP with a CRMP2 antibody or normal IgG antibody. Naringenin was detected abundantly 


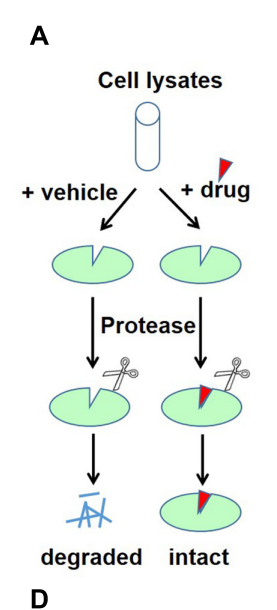

D

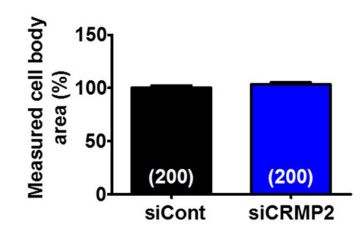

E

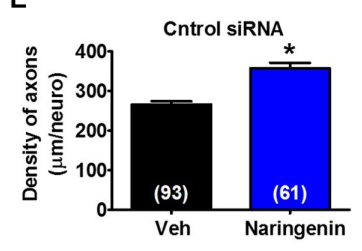

B

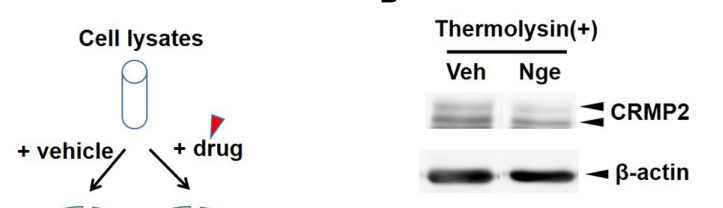

C

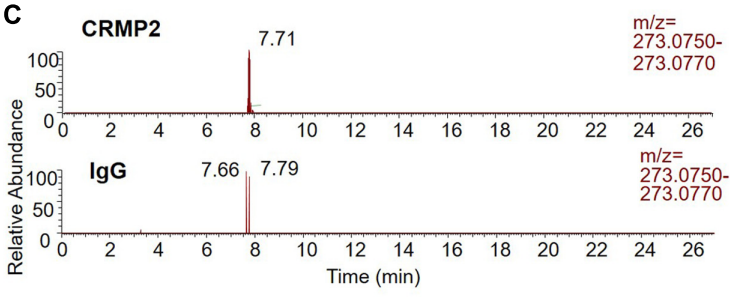

$\mathbf{F}$
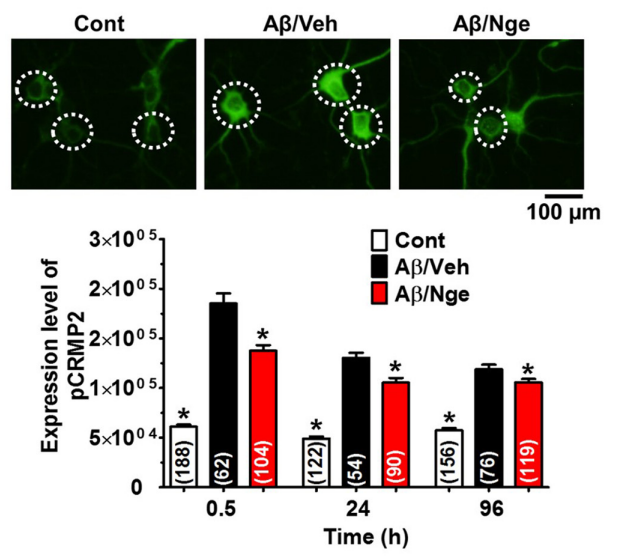

FIGURE 6 | CRMP2 is a target protein of naringenin and is involved in naringenin-induced axonal regrowth. (A) Illustration of the DARTS method. (B) Identification of CRMP2 as a target of naringenin by DARTS and western blot. Mouse cortical neurons were lysed, naringenin or vehicle solution was incubated with lysates for $1 \mathrm{~h}$ at room temperature. The mixture was proteolysed using thermolysin and electrophoresed for western blot [Student's unpaired $t$-test, ${ }^{*} p<0.05$ vs. Veh (T+). The number of repeated experiments is shown in each column], full-length blots are presented in Supplementary Figure 9. (C) Confirmation of the binding between naringenin and CRMP2 by IP coupled with LC-MS analysis. Primary cultured neurons were maintained for 3 days and then treated with naringenin for $6 \mathrm{~h}$. Cell lysates were immunoprecipitated with a CRMP2 (Upper) or normal lgG (Lower) antibody, and the pellets were extracted with methanol and subjected to LC-MS. (D) Three days after transfection with siCRMP2 $(1 \mu \mathrm{M})$ and GFP vector $(2 \mu \mathrm{g})$, the neurons were stained for a CRMP2 antibody. The level of CRMP2 in the GFP-positive neurons was quantified. (E) CRMP2 siRNA $(1 \mu \mathrm{M})$ or control siRNA $(1 \mu \mathrm{M})$ with GFP vector was transfected into cortical cells. Two days later, the cells were immunostained for pNF-H after treated with $10 \mu \mathrm{M}$ naringenin or vehicle $(0.1 \% \mathrm{DMSO})$ for 4 days. The pNF-H-positive axons in the GFP-positive neurons were quantified. (F) Naringenin treatment decreased the A $\beta$-induced phosphorylation of CRMP2. Cortical neurons were cultured for 3 days, treated with $A \beta_{25}-35$ or vehicle solution $\left(\mathrm{dH}_{2} \mathrm{O}\right)$ for another 3 days, and then treated with naringenin $(1 \mu \mathrm{M})$ or vehicle $(0.1 \% \mathrm{DMSO})$ for 0.5 (representative photos), 24 , or $96 \mathrm{~h}$ after the removal of the A $\beta$-containing medium. The cells were double immunostained for pCRMP2 and MAP2. The level of pCRMP2 in neurons was quantified. (D-F) The number of neurons is shown in each column. Student's unpaired $t$-tests were performed in (D,E) $\left({ }^{*} p<0.05\right)$, and one-way ANOVA with Dunnett's post hoc test was performed in (F) ${ }^{*} p<0.05$ vs. A $/$ Neh).

after IP with the CRMP2 antibody (peak area: 169,940) but was rarely detected in the IgG immunoprecipitated sample (peak area: 8,265 ) (Figure 6C). The single peak detected in the group without the CRMP2 antibody might be caused by a small amount of non-specific binding between protein $\mathrm{G}$ and the CRMP2 protein (Supplementary Figure 7C).

To investigate whether CRMP2 mediates naringenin-induced axonal extension, CRMP2 was knocked down using siRNA transfection. Three days after the transfection of siRNA targeting CRMP2 (1 $\mu \mathrm{M})$, the CRMP2 level in the GFP-transfected neurons was significantly reduced (44\%) compared with the control siRNA-transfected neurons (Figure 6D, Right). There were no changes in the measured cell body area (Figure 6D, Left). In control siRNA-transfected neurons, the axon density was significantly increased by naringenin treatment (Figure 6E). However, the naringenin-induced axonal growth was completely abolished by transfection of CRMP2 siRNA (Figure 6E). These data indicate that CRMP2 is a target of naringenin and is essential for naringenin-induced axonal growth.

CRMP2 is critical for the regulation of neurite outgrowth due to its binding to tubulin heterodimers (Fukata et al., 2002). Hyperphosphorylated CRMP2 is involved in the A $\beta$-induced impairment of cognitive memory and is characteristic of Alzheimer's disease (Gu et al., 2000; Cole et al., 2007; Soutar 
et al., 2009; Isono et al., 2013). CRMP2 is phosphorylated by Cdk 5 at Ser522 and subsequently phosphorylated by GSK$3 \beta$ at Ser518, Thr514, and Thr509 (Uchida et al., 2005). Therefore, we investigated the effect of naringenin on the expression of pThr514-CRMP2 in A $\beta$-treated primary cultured cortical neurons. Compared with the control group, CRMP2 was hyperphosphorylated at Thr514 after $\mathrm{A} \beta_{25-35}$ treatment; the pCRMP2 level decreased significantly after naringenin treatment (Figure 6F).

Collectively, our findings revealed that naringenin binds to CRMP2 and reduces the $A \beta$-induced phosphorylation of CRMP2, resulting in axonal growth facilitation.

\section{DISCUSSION}

Generally speaking, it is challenging and time-consuming to isolate, identify, and screen the bioactive components in natural medicines. In addition, the components may be metabolized to phase I and II derivatives following absorption. Therefore, the bioavailability and access of metabolites or natural components to brain tissues are absolutely critical factors in predicting the potential for those compounds as CNS drugs. Thus, studies investigating the bioactivity and physiological mechanisms of natural medicines must use the metabolites that have been identified in the target tissues. Here, we report a new strategy that couples biochemical analysis with pharmacological methods and can be used to evaluate therapeutic candidates for $\mathrm{AD}$ in natural medicines. Using this method, we found that a crude drug, DR, ameliorated memory deficits and AD-like pathologies in 5XFAD model mice. To clarify the mechanism of action for $\mathrm{DR}$ in the treatment of $\mathrm{AD}$, we first identified metabolites that were delivered into the brain. To detect enough concentration and a variety of metabolites as possible as we can, we administered a single dose of DR (13 g $\mathrm{kg}^{-1}$ ) to the mice. As a result, only 3 metabolites were identified in the brain cortex with comprehensive FT-MS-MS analysis.

Previously, we identified five main compounds in the water extract of DR using bioassay-guided isolation, with two of them (neoeriocitrin and caffeic acid-4-O-glucoside) showing significant axonal elongation effects after $A \beta_{25-35}$-induced atrophy (Yang et al., 2015). In the present study, these two compounds were detected in the blood but not in the brain after the oral administration of DR (Supplementary Table 1), demonstrating that they could not penetrate the BBB and enter into the brain. Naringin did not show axonal regrowth activity and was not detected in the brain. Nonetheless, two metabolites of naringin-naringenin and naringenin-7-O-glucuronide-were found in the brain after the oral administration of DR, and these metabolites reversed $A \beta$-induced axonal atrophy. Here, we directly identified the metabolites delivered into the brain after an oral dose of a crude extract. Therefore, this method avoids the identification of false-positive compounds that occurs with standard bioassay-guided isolation methods and may be a fast, economical, and efficient way to identify effective metabolites for use as interventions for AD.
Glucuronidation mediated by UDP-glucuronosyltransferases (UGTs) is an important metabolic pathway that facilitates the clearance of xenobiotics, including drugs and environmental substances, and functions in the metabolism of endogenous compounds (Rowland et al., 2013). These hydrophilic glucuronides are generally inactive and show poor BBB permeability, while some glucuronides, such as morphine6-glucuronide and quercetin-3-O-glucuronide, exist in the brain and show important biological activities (Ho et al., 2013; Klimas and Mikus, 2014). Our present report also shows that naringenin-7-O-glucuronide can penetrate the $\mathrm{BBB}$ and enhance axonal regrowth. How do these glucuronides exist in the brain? One possible explanation is the existence of a transporter at the $\mathrm{BBB}$. The organic anion transporter (OAT) is localized at the membrane of $\mathrm{BBB}$ cells and is responsible for the transportation of numerous endogenous and xenobiotic amphipathic compounds (Kullak-Ublick et al., 2001; Sugiyama et al., 2003; Ohtsuki et al., 2004). Moreover, OAT can actively transport morphine-6-glucuronide across the BBB (Bourasset et al., 2003). However, there are no reports regarding a naringenin-glucuronide transporter. Another explanation is the finding of UGTs in the brain and neurons. In the brain, UGTs are mainly expressed in endothelial cells and astrocytes of the BBB (Ouzzine et al., 2014). The expression of glucuronosyltransferase isoform UGT1A6 was reported in primary cultures of neurons and astrocytes (Suleman et al., 1998). Our results also provide evidence that UGTs are present in neurons (Supplementary Figure 7A). Thus, it is possible that naringenin was absorbed by neurons and glucuronidated by UGTs after penetrating the BBB.

Drug affinity responsive target stability has been used for the identification of ligand targets by identifying the alterations in proteolytic sensitivity that occur after the binding of ligand and receptor (Lomenick et al., 2009). Therefore, this method might be used to identify new targets for the treatment of AD. Here, we first combined FT-MS-MS with the DARTS method to discover a brain-penetrating compound, naringenin, and its target protein CRMP2. Actually, DARTS is based on the conformation changes after drug binding, resulting in a decrease or an increase in proteolytic susceptibility of the target protein (Lomenick et al., 2009). In this study, DR showed increased band density while naringenin showed decreased band density of CRMP2 compared with vehicle treated thermolysin group in DARTS analysis. This might due to non-specifically binding of DR derived components (except for naringenin) with CRMP2 in case of DARTS used DR extract, because the proteolytic susceptibility of CRMP2 might be changed compared with that of CRMP2 induced by naringenin. Since naringenin-7-O-glucuronide showed axonal regrowth activity after $A \beta$-induced axonal atrophy, it is possible that naringenin-7$O$-glucuronide binds to CRMP2. Thus, we performed the DARTS experiment of naringenin and its glucuronides using lysed neurons. The result was shown in Supplementary Figure 8, except for naringenin, naringenin-7-O-glucuronide but not naringenin$4^{\prime}$-O-glucuronide also showed the possible binding possibility to CRMP2. This was corresponding to the in vitro assay result, 
which indicated that naringenin-induced cellular signaling was attributed to naringenin and naringenin-7-O-glucuronide.

Naringenin has been reported to improve learning and memory in $\mathrm{A} \beta$ or streptozotocin-injected $\mathrm{AD}$ models (Yang et al., 2014; Ghofrani et al., 2015), which might relate to the mitigation of lipid peroxidation and apoptosis or the increased insulin and insulin receptor expression in the rat brain. Naringenin also showed anti-inflammatory activity in LPS/IFN- $\gamma$-stimulated glial cells and neuroprotective effects against $\mathrm{H}_{2} \mathrm{O}_{2}$-induced hyper-oxidation (Heo et al., 2004; Vafeiadou et al., 2009). In the present study, we found that naringenin enhanced axonal growth in both normal neurons and neurons subjected to $A \beta$-induced axonal atrophy. This growth might contribute to the reconstruction of neural networks and improvements in memory. With the exception of lacosamide, which reduced the association between Cdk5phosphorylated CRMP2 and CaV2.2 in vitro and in vivo (Moutal et al., 2016), the binding of small molecules to CRMP2 has rarely been reported. Moreover, lacosamide inhibited CRMP2-dependent tubulin polymerization, which prevents the growth of neurites. The present study is the first to describe a molecule, naringenin that targets CRMP2 and inhibits CRMP2 phosphorylation at Thr514. This might restore the association between CRMP2 and tubulin, leading to axonal regrowth.

In summary, we first reported that DR enhances memory function and ameliorates $\mathrm{AD}$ pathologies in 5XFAD mice. LTQ-Orbitrap-FT-MS-MS and DARTS analysis pinpointed the effective substances in DR and identified a molecule that binds to CRMP2. This systematic strategy might contribute to the development of natural medicines and exploration of

\section{REFERENCES}

Anuja, G. I., Latha, P. G., Suja, S. R., Shyamal, S., Shine, V. J., Sini, S., et al. (2010). Anti-inflammatory and analgesic properties of Drynaria quercifolia (L.) J. Smith. J. Ethnopharmacol. 132, 456-460. doi: 10.1016/j.jep.2010.08.038

Bourasset, F., Cisternino, S., Temsamani, J., and Scherrmann, J. M. (2003). Evidence for an active transport of morphine-6-beta-d-glucuronide but not P-glycoprotein-mediated at the blood-brain barrier. J. Neurochem. 86, 1564-1567. doi: 10.1046/j.1471-4159.2003.01990.x

Cole, A. R., Noble, W., Aalten, L., Plattner, F., Meimaridou, R., Hogan, D., et al. (2007). Collapsin response mediator protein-2 hyperphosphorylation is an early event in Alzheimer's disease progression. J. Neurochem. 103, 1132-1144. doi: 10.1111/j.1471-4159.2007.04829.x

Dere, E., Huston, J. P., and De-Souza-Silva, M. A. (2005). Integrated memory for objects, places, and temporal order: evidence for episodic-like memory in mice. Neurobiol. Learn. Mem. 84, 214-221. doi: 10.1016/j.nlm.2005.07.002

Dickson, T. C., and Vickers, J. C. (2001). The morphological phenotype of $\beta$-amyloid plaques and associated neuritic changes in Alzheimer's disease. Neuroscience 105, 99-107. doi: 10.1016/S0306-4522(01)00169-5

Durairajan, S. S., Liu, L. F., Lu, J. H., Chen, L. L., Yuan, Q., Chung, S. K., et al. (2012). Berberine ameliorates $\beta$-amyloid pathology, gliosis, and cognitive impairment in an Alzheimer's disease transgenic mouse model. Neurobiol. Aging 33, 2903-2919. doi: 10.1016/j.neurobiolaging.2012.02.016

Fang, T., Wang, Y., Ma, Y., Su, W., Bai, Y., and Zhao, P. (2006). A rapid LC/MS/MS quantitation assay for naringin and its two metabolites in rats plasma. J. Pharm. Biomed. Anal. 40, 454-459. doi: 10.1016/j.jpba.2005.07.031

Fujiwara, H., Takayama, S., Iwasaki, K., Tabuchi, M., Yamaguchi, T., Sekiguchi, K., et al. (2011). Yokukansan, a traditional Japanese medicine, ameliorates memory disturbance and abnormal social interaction with anti-aggregation effect of their mechanisms. Moreover, this strategy might be broadly applicable to the identification of novel compounds and targets for intervention in CNS diseases.

\section{AUTHOR CONTRIBUTIONS}

ZY, TK, and CT designed the experiments and wrote the manuscript. ZY performed the experiments and analyzed the data. CT supervised all experiments and analyses.

\section{FUNDING}

This work was supported by a grant-in-aid for the Cooperative Research Project from the Institute of Natural Medicine at the University of Toyama in 2014 and 2015 and the Discretionary Funds of the President of the University of Toyama.

\section{ACKNOWLEDGMENT}

Dr. Kohei Kazuma (University of Toyama) provided advice on the LC-MS analysis.

\section{SUPPLEMENTARY MATERIAL}

The Supplementary Material for this article can be found online at: http://journal.frontiersin.org/article/10.3389/fphar. 2017.00340/full\#supplementary-material

cerebral amyloid $\beta$ proteins in amyloid precursor protein transgenic mice. Neuroscience 180, 305-313. doi: 10.1016/j.neuroscience.2011.01.064

Fukata, Y., Itoh, T. J., Kimura, T., Ménager, C., Nishimura, T., Shiromizu, T., et al. (2002). CRMP-2 binds to tubulin heterodimers to promote microtubule assembly. Nat. Cell Biol. 4, 583-591. doi: 10.1038/ncb825

Ghofrani, S., Joghataei, M. T., Mohseni, S., Baluchnejadmojarad, T., Bagheri, M., Khamse, S., et al. (2015). Naringenin improves learning and memory in an Alzheimer's disease rat model: insights into the underlying mechanisms. Eur. J. Pharmacol. 764, 195-201. doi: 10.1016/j.ejphar.2015.07.001

Gu, Y., Hamajima, N., and Ihara, Y. (2000). Neurofibrillary tangle-associated collapsin response mediator protein-2 (CRMP-2) is highly phosphorylated on Thr-509, Ser-518, and Ser-522. Biochemistry 39, 4267-4275. doi: 10.1021/ bi992323h

Heo, H. J., Kim, D. O., Shin, S. C., Kim, M. J., Kim, B. G., and Shin, D. H. (2004). Effect of antioxidant flavanone, naringenin, from Citrus junos on neuroprotection. J. Agric. Food Chem. 52, 1520-1525. doi: 10.1021/jf03 $5079 \mathrm{~g}$

Ho, L., Ferruzzi, M. G., Janle, E. M., Wang, J., Gong, B., Chen, T. Y., et al. (2013). Identification of brain-targeted bioactive dietary quercetin-3-O-glucuronide as a novel intervention for Alzheimer's disease. FASEB J. 27, 769-781. doi: 10.1096/ fj.12-212118

Isono, T., Yamashita, N., Obara, M., Araki, T., Nakamura, F., Kamiya, Y., et al. (2013). Amyloid- $\beta 25-35$ induces impairment of cognitive function and longterm potentiation through phosphorylation of collapsin response mediator protein 2. Neurosci. Res. 77, 180-185. doi: 10.1016/j.neures.2013.08.005

Klimas, R., and Mikus, G. (2014). Morphine-6-glucuronide is responsible for the analgesic effect after morphine administration: a quantitative review of morphine, morphine-6-glucuronide, and morphine-3-glucuronide. Br. J. Anaesth. 113, 935-944. doi: 10.1093/bja/aeu186 
Koehn, F. E., and Carter, G. T. (2005). The evolving role of natural products in drug discovery. Nat. Rev. Drug Discov. 4, 206-220. doi: 10.1038/nrd1657

Kuboyama, T., Lee, Y. A., Nishiko, H., and Tohda, C. (2015). Inhibition of clathrinmediated endocytosis prevents amyloid $\beta$-induced axonal damage. Neurobiol. Aging 36, 1808-1819. doi: 10.1016/j.neurobiolaging.2015.02.005

Kuboyama, T., Tohda, C., and Komatsu, K. (2005). Neuritic regeneration and synaptic reconstruction induced by withanolide A. Br. J. Pharmacol. 144, 961-971. doi: 10.1038/sj.bjp.0706122

Kullak-Ublick, G. A., Ismair, M. G., Stieger, B., Landmann, L., Huber, R., Pizzagalli, F., et al. (2001). Organic anion-transporting polypeptide B (OATP-B) and its functional comparison with three other OATPs of human liver. Gastroenterology 120, 525-533. doi: 10.1053/gast.2001.21176

Kushida, H., Fukutake, M., Tabuchi, M., Katsuhara, T., Nishimura, H., Ikarashi, Y., et al. (2013). Simultaneous quantitative analyses of indole and oxindole alkaloids of Uncaria Hook in rat plasma and brain after oral administration of the traditional Japanese medicine Yokukansan using high-performance liquid chromatography with tandem mass spectrometry. Biomed. Chromatogr. 27, 1647-1656. doi: 10.1002/bmc.2974

Lee, Y. E., Liu, H. C., Lin, Y. L., Liu, S. H., Yang, R. S., and Chen, R. M. (2014). Drynaria fortunei J. Sm. improves the bone mass of ovariectomized rats through osteocalcin-involved endochondral ossification. J. Ethnopharmacol. 158, 94-101. doi: 10.1016/j.jep.2014.10.016

Lomenick, B., Hao, R., Jonai, N., Chin, R. M., Aghajan, M., Warburton, S., et al. (2009). Target identification using drug affinity responsive target stability (DARTS). Proc. Natl. Acad. Sci. U.S.A. 106, 21984-21989. doi: 10.1073/pnas. 0910040106

Moutal, A., François-Moutal, L., Perez-Miller, S., Cottier, K., Chew, L. A., Yeon, S. K., et al. (2016). (S)-Lacosamide binding to collapsin response mediator protein 2 (CRMP2) regulates CaV2.2 activity by subverting its phosphorylation by Cdk5. Mol. Neurobiol. 53, 1959-1976. doi: 10.1007/s12035-0159141-2

Niu, Y., Li, F., Inada, C., Tanaka, K., Watanabe, S., Fujiwara, H., et al. (2015). Chemical profiling with HPLC-FTMS of exogenous and endogenous chemicals susceptible to the administration of chotosan in an animal model of type 2 diabetes-induced dementia. J. Pharm. Biomed. Anal. 104, 21-30. doi: 10.1016/j. jpba.2014.11.019

Ohtsuki, S., Kikkawa, T., Mori, S., Hori, S., Takanaga, H., Otagiri, M., et al. (2004). Mouse reduced in osteosclerosis transporter functions as an organic anion transporter 3 and is localized at abluminal membrane of bloodbrain barrier. J. Pharmacol. Exp. Ther. 309, 1273-1281. doi: 10.1124/jpet.103. 063370

Ouzzine, M., Gulberti, S., Ramalanjaona, N., Magdalou, J., and Fournel-Gigleux, S. (2014). The UDP-glucuronosyltransferases of the blood-brain barrier: their role in drug metabolism and detoxication. Front. Cell. Neurosci. 8:349. doi: 10.3389/ fncel.2014.00349

Pai, M. Y., Lomenick, B., Hwang, H., Schiestl, R., McBride, W., Loo, J. A., et al. (2015). Drug affinity responsive target stability (DARTS) for small-molecule target identification. Methods Mol. Biol. 1263, 287-298. doi: 10.1007/978-14939-2269-7_22

Rowland, A., Miners, J. O., and Mackenzie, P. I. (2013). The UDPglucuronosyltransferases: their role in drug metabolism and detoxification. Int. J. Biochem. Cell Biol. 45, 1121-1132. doi: 10.1016/j.biocel.2013.02.019

Shigyo, M., Kuboyama, T., Sawai, Y., Tada-Umezaki, M., and Tohda, C. (2015). Extracellular vimentin interacts with insulin-like growth factor 1 receptor to promote axonal growth. Sci. Rep. 5:12055. doi: 10.1038/srep12055

Soutar, M. P., Thornhill, P., Cole, A. R., and Sutherland, C. (2009). Increased CRMP2 phosphorylation is observed in Alzheimer's disease; does this tell us anything about disease development? Curr. Alzheimer Res. 6, 269-278. doi: $10.2174 / 156720509788486572$
Sugiyama, D., Kusuhara, H., Taniguchi, H., Ishikawa, S., Nozaki, Y., Aburatani, H., et al. (2003). Functional characterization of rat brain-specific organic anion transporter (Oatp14) at the blood-brain barrier: high affinity transporter for thyroxine. J. Biol. Chem. 278, 43489-43495. doi: 10.1074/jbc.M306933200

Suleman, F. G., Abid, A., Gradinaru, D., Daval, J. L., Magdalou, J., and Minn, A. (1998). Identification of the uridine diphosphate glucuronosyltransferase isoform UGT1A6 in rat brain and in primary cultures of neurons and astrocytes. Arch. Biochem. Biophys. 358, 63-67. doi: 10.1006/abbi.1998.0842

Tan, X. S., Ma, J. Y., Feng, R., Ma, C., Chen, W. J., Sun, Y. P., et al. (2013). Tissue distribution of berberine and its metabolites after oral administration in rats. PLOS ONE 8:e77969. doi: 10.1371/journal.pone.0077969

Tohda, C., Matsumoto, N., Zou, K., Meselhy, M. R., and Komatsu, K. (2004). Abeta(25-35)-induced memory impairment, axonal atrophy, and synaptic loss are ameliorated by M1, A metabolite of protopanaxadiol-type saponins. Neuropsychopharmacology 29, 860-868. doi: 10.1038/sj.npp.1300388

Tohda, C., Urano, T., Umezaki, M., Nemere, I., and Kuboyama, T. (2012). Diosgenin is an exogenous activator of 1,25D3-MARRS/Pdia3/ERp57 and improves Alzheimer's disease pathologies in 5XFAD mice. Sci. Rep. 2:535. doi: 10.1038/srep00535

Uchida, Y., Ohshima, T., Sasaki, Y., Suzuki, H., Yanai, S., Yamashita, N., et al. (2005). Semaphorin 3 A signaling is mediated via sequential Cdk5 and GSK3beta phosphorylation of CRMP2: implication of common phosphorylating mechanism underlying axon guidance and Alzheimer's disease. Genes Cells 10, 165-179. doi: 10.1111/j.1365-2443.2005.00827.x

Vafeiadou, K., Vauzour, D., Lee, H. Y., Rodriguez-Mateos, A., Williams, R. J., and Spencer, J. P. (2009). The citrus flavanone naringenin inhibits inflammatory signaling in glial cells and protects against neuroinflammatory injury. Arch. Biochem. Biophys. 484, 100-109. doi: 10.1016/j.abb.2009.01.016

Wang, J., Ferruzzi, M. G., Ho, L., Blount, J., Janle, E. M., Gong, B., et al. (2012). Brain-targeted proanthocyanidin metabolites for Alzheimer's disease treatment. J. Neurosci. 32, 5144-5150. doi: 10.1523/JNEUROSCI.6437-11.2012

Wishart, D. S. (2016). Emerging applications of metabolomics in drug discovery and precision medicine. Nat. Rev. Drug Discov. 15, 473-484. doi: 10.1038/nrd. 2016.32

Xiang, C., Qiao, X., Wang, Q., Li, R., Miao, W., Guo, D., et al. (2011). From single compounds to herbal extract: a strategy to systematically characterize the metabolites of licorice in rats. Drug Metab. Dispos. 39, 1597-1608. doi: $10.1124 / \mathrm{dmd} .111 .038695$

Xiao, J. F., Zhou, B., and Ressom, H. W. (2012). Metabolite identification and quantitation in LC-MS/MS-based metabolomics. Trends Analyt. Chem. 32, 1-14. doi: 10.1016/j.trac.2011.08.009

Yang, W., Ma, J., Liu, Z., Lu, Y., Hu, B., and Yu, H. (2014). Effect of naringenin on brain insulin signaling and cognitive functions in ICV-STZ induced dementia model of rats. Neurol. Sci. 35, 741-751. doi: 10.1007/s10072-013-1594-3

Yang, Z. Y., Kuboyama, T., Kazuma, K., Konno, K., and Tohda, C. (2015). Active Constituents from Drynaria fortunei rhizomes on the attenuation of $A \beta(25-35)$ induced axonal atrophy. J. Nat. Prod. 78, 2297-2300. doi: 10.1021/acs.jnatprod. $5 \mathrm{~b} 00290$

Conflict of Interest Statement: The authors declare that the research was conducted in the absence of any commercial or financial relationships that could be construed as a potential conflict of interest.

Copyright (c) 2017 Yang, Kuboyama and Tohda. This is an open-access article distributed under the terms of the Creative Commons Attribution License (CC BY). The use, distribution or reproduction in other forums is permitted, provided the original author(s) or licensor are credited and that the original publication in this journal is cited, in accordance with accepted academic practice. No use, distribution or reproduction is permitted which does not comply with these terms. 